\title{
An input-independent method for solving weakly nonlinear partial differential equations in the frequency domain: application to the Euler equations
}

\author{
David Quero* \\ DLR (German Aerospace Center), Institute of Aeroelasticity, Göttingen, Germany
}

\begin{abstract}
In this work a novel approach in determining the first and second order frequencydomain Volterra kernels for weakly nonlinear partial differential equations (PDEs) in semidiscrete form based on the application of the harmonic probing (HP) method is presented. This represents a formal extension of the linearized-frequency domain (LFD) methods to a nonlinear framework, leading to a so-called LFD2 method. The method allows for the representation of weak nonlinearities by solving two input-independent linear algebraic systems of equations in the frequency domain and thus circumvents the solution of the nonlinear PDE by numerical integration for each different input, representing a nonlinear reduced-order model (ROM) for the physical phenomena. The general form of the equations is derived and an application to the well known viscous Burgers' equation to show its suitability in representing the nonlinear convective term is shown. Next, an application to the compressible quasi one-dimensional unsteady flow described by the Euler equations is presented. The proposed method overcomes two constraints present in other methods for the solution of nonlinear PDEs, namely, the consideration of exclusively periodic solutions as in the harmonic balance (HB) method and the dependency of the kernels with the input signal as in the Volterra kernel identification methods.
\end{abstract}

\section{Introduction}

In the recent years there has been a growing interest in the area of approximation of nonlinear largescale systems described by partial differential equations (PDEs), leading to a large number of reduced-order models (ROMs) which aim at obtaining a very similar physical description to that provided by the original PDE but at much lower computational cost.

A popular approach in the aerodynamic and aeroelasticity fields is the linearization of the PDE equations describing the flow around a reference state, which has shown its effectiveness for aeroelastic stability and dynamic response prediction $1[2]$ Other approaches for the generation of linearized ROMs include the eigensystem realization algorithm (ERA) ${ }^{3}$ or the Loewner approach ${ }^{[4}$ However, the linearized approach presents limitations in cases involving the presence of large shock motions or separation areas and the development of limit cycle oscillations (LCO). The linearized (also known as dynamically linear) approach is equivalent to a first order Volterra functional series expansion of the governing PDE in the incremental variables.

Especially in the field of aeroelasticity the determination of ROMs which are able to describe nonlinear phenomena for a range of input signals is of great relevance. An overview of different aerodynamic and aeroelastic ROMs are provided by Lucia et al.$^{5}$ and Ghoreyshi et al ${ }^{[6}$ They include the proper orthogonal decomposition (POD) $\sqrt[7]{7 \sqrt[8]{8}}$ harmonic balance (HB) method, ${ }^{[11}$ neural networks $\sqrt{12}[13$ and the Volterra series. On the one hand, an extension of the POD method denoted as discrete empirical interpolation method (DEIM) for nonlinear applications has been applied to aerodynamic ${ }^{14}$ and aeroelastic models ${ }^{15}$ The PODDEIM method is a data-driven approach and as such requires the generation of a training dataset. Also, the predicted trajectories must remain close to the ones used in the training set.

On the other hand, the Volterra functional series expansion is valid for weak nonlinearities caused by input signals of arbitrary shape provided they remain small. An extensive review of the applications of the

\footnotetext{
${ }^{*}$ Research Scientist, Bunsenstraße 10, Göttingen.
} 
Volterra series to different engineering disciplines including mechanical, control, electrical and aeroelastic systems is provided by Cheng et al ${ }^{16}$ Several aeroelastic applications of the Volterra theory have been presented ${ }^{17}$ Silva ${ }^{18}$ demonstrated the identification of the Volterra kernels of first and second order of the Burger's viscous equation as a representative case of the nonlinear convective phenomena. In the same work Silva identified the Volterra kernels corresponding to the unsteady flow around an airfoil described by the Euler and Reynolds-averaged Navier-Stokes (RANS) equations. Balajewicz et al ${ }^{[19}$ used sparse or pruned Volterra series to model the inviscid unsteady compressible flow aver a NACA0012 airfoil and the LCOs induced by the inviscid unsteady aerodynamic nonlinearities.20 ${ }^{20}$ Marzocca et al ${ }^{21}$ analytically determined the Volterra kernels corresponding to a two-dimensional aeroelastic system in linear unsteady incompressible flow with nonlinear stiffness and damping terms in the heave and pitch degrees of freedom of the structure. In connection to the Volterra series expansion for nonlinear systems, the ERA has been used for the identification of nonlinear systems by a bilinear model,$[22$ but no application to aerodynamic or aeroelastic systems has been done yet.

One of the advantages of the Volterra theory is the corresponding description in the frequency domain, allowing for an extension of the well known concept of transfer function matrices to the higher order kernels representing the nonlinear effects. This work aims at extending the linearized approach of flow phenomena with the inclusion of (weak) nonlinearities after employing a second order Volterra functional series expansion in the incremental variables. This extension turns out to be very efficient as the kernels can be computed with two linear algebraic systems of equations which are independent of the input. The nonlinearity is thus shifted to the functional series expansion, providing a nonlinear ROM description of the weakly nonlinear PDE valid for arbitrary inputs. To the best of the author's knowledge, up to now only identification techniques have been applied for the determination of the Volterra kernels corresponding to the nonlinear compressible flow equations. In this work an extension of the harmonic probing (HP) method of Worden et al ${ }^{23}$ is presented which allows for the computation of the Volterra kernels corresponding to the nonlinear flow equations directly and overcoming the use of identification techniques. This formal extension of the existing linearized frequency-domain (LFD) method is then called linearized frequency-domain method for a second order functional series expansion (LFD2) in this work. The term linearized frequency-domain refers to the fact that only linear algebraic solvers in the frequency domain are required for the proposed LFD2 method, even though it is able to consider nonlinearities present in the original PDE.

Note that unlike for the harmonic balance (HB) method, the nonlinear frequency domain description of the nonlinear ROM obtained in this work is valid for arbitrary inputs provided the system nonlinearities are weak. A second order expansion is considered in this work and for higher nonlinearities additional terms must be included in the functional series expansion. The HB technique is able to describe larger nonlinearities by considering a higher number of harmonics (with a corresponding increase of computational effort) at the expense of an input dependency.

Apart from the validity of the presented nonlinear ROM to arbitrary excitations, the proposed method presents fundamental differences when compared to other existing methods:

- A functional series expansion instead of a (Fourier) function series expansion is taken into account. This means that the LFD2 method presented here is valid for weak nonlinearities around the initial state in equilibrium condition. Compared to other methods for the solution of nonlinear PDEs such as the perturbation and Adomian methods or the more general homotopy analysis method, ${ }^{24}$ the LFD2 method does not require a solution sequence for each different input ${ }^{25]}$ but is limited to the existence of exclusively weak nonlinearities.

- The proposed method is simulation-free and as such training data need not be generated numerically, avoiding the repeated solution of the nonlinear PDE for different datasets.

- Unlike the Volterra kernel identification methods, the computation of the Volterra kernels is independent of the input amplitude, see Section III.A.

- Even for harmonic inputs, the transient response is captured. If the transient behaviour for harmonic excitations is of no interest, a very efficient implementation can be used to compute the steady-state harmonic response, see Section III.B

Applications to the Burgers' viscous equation and to the quasi-one dimensional unsteady compressible flow described by the Euler equations are provided in Section IV. 


\section{Functional series}

For a single-input analytic system the output component $j=1, \ldots, N$ of the output $\mathbf{W}(t) \in \mathbb{R}^{N}$ for $t \geq 0$ can be expressed as a Volterra or functional series expansion, $\frac{18}{1}$

$$
W_{j}(t)=\sum_{n=1}^{\infty} \int_{-\infty}^{\infty} h_{n}^{(j)}\left(\tau_{1}, \ldots, \tau_{n}\right) \prod_{k=1}^{n} u\left(t-\tau_{k}\right) d \tau_{k}
$$

where $h_{n}^{(j)}\left(\tau_{1}, \ldots, \tau_{n}\right) \in \mathbb{R}$ represents the Volterra kernel of order $n$ for the output component $W_{j}(t)$ and $u(t)$ is the input signal. Note that the Volterra kernels are independent of the input $u(t)$. When the governing equation of the system is known the harmonic probing (HP) technique can be applied, as done by Worden et al ${ }^{23}$ for the determination of the Volterra kernels in the frequency domain for mechanical systems represented by ordinary differential equations (ODEs).

The Volterra or functional series expansion has an equivalent counterpart description in the frequency domain. The frequency-domain Volterra kernel $H_{n}^{(j)}\left(\omega_{1}, \ldots, \omega_{n}\right)$ of order $n$ relates to the Volterra kernel $h_{n}^{(j)}\left(\tau_{1}, \ldots, \tau_{n}\right)$ by a multidimensional Fourier transform,

$$
H_{n}^{(j)}\left(\omega_{1}, \ldots, \omega_{n}\right)=\int_{-\infty}^{\infty} \ldots \int_{-\infty}^{\infty} h_{n}^{(j)}\left(\tau_{1}, \ldots, \tau_{n}\right) e^{-i\left(\omega_{1} \tau_{1}+\ldots+\omega_{n} \tau_{n}\right)} d \tau_{1} \ldots d \tau_{n}
$$

In this work the HP method is extended for general single-input systems represented by a nonlinear PDE, obtaining the Volterra kernels of first and second order in the frequency domain. The numerical implementation of a third order functional series expansion has been shown computationally very costly. Instead, Balajewicz et al $\sqrt[19[20]{[2} \text { successfully applied a pruned or sparse Volterra series of third order to }$ aerodynamic and aeroelastic systems. Even though a third order functional series expansion has been shown adequate for an aeroelastic system with structural nonlinearities, ${ }^{21}$ in Section IV is shown that a second order functional series expansion is able to describe the weak nonlinear behaviour of the convective terms arising in the PDE describing the flow phenomena.

Once the Volterra kernels are known the system output $\mathbf{W}(t)$ can be readily obtained. In particular, for a second order functional series expansion the output component $W_{j}(t)$ is given by:

$$
W_{j}(t)=\int_{-\infty}^{\infty} h_{1}^{(j)}\left(\tau_{1}\right) u\left(t-\tau_{1}\right) d \tau_{1}+\int_{-\infty}^{\infty} \int_{-\infty}^{\infty} h_{2}^{(j)}\left(\tau_{1}, \tau_{2}\right) u\left(t-\tau_{1}\right) u\left(t-\tau_{2}\right) d \tau_{1} d \tau_{2} .
$$

The frequency counterpart can be obtained by:

$$
W_{j}(\omega)=H_{1}^{(j)}(\omega) u(\omega)+\frac{1}{2 \pi} \int_{-\infty}^{\infty} H_{2}^{(j)}\left(\omega_{1}, \omega-\omega_{1}\right) u\left(\omega_{1}\right) u\left(\omega-\omega_{1}\right) d \omega_{1},
$$

where $W_{j}(\omega)$ is the Fourier transform of the signal $W_{j}(t)$ and the Volterra kernels in the time and frequency domain are related by uni- $(n=1)$ and bidimensional $(n=2)$ Fourier transforms, see Eq. 2 .

\section{Harmonic probing for PDEs}

The HP method for ODEs as presented by Worden et al ${ }^{23}$ is based on the system response to a multi-tone (complex) input ${ }^{26}$ For the determination of the first and second order kernels the response to a single- and two-tone inputs is required. The response to a single input provides the first order kernel, which coincides with the transfer function of the linearized system. The second order kernel can be obtained by considering the output to a two-tone input signal:

$$
u(t)=A_{1} e^{i \omega_{1} t}+A_{2} e^{i \omega_{2} t},
$$

and neglecting the contribution of higher order kernels: 


$$
\begin{aligned}
W_{j}(t) & =H_{1}^{(j)}\left(\omega_{1}\right) A_{1} e^{i \omega_{1} t}+H_{1}^{(j)}\left(\omega_{2}\right) A_{2} e^{i \omega_{2} t} \\
& +H_{2}^{(j)}\left(\omega_{1}, \omega_{1}\right) A_{1}^{2} e^{i 2 \omega_{1} t}+2 H_{2}^{(j)}\left(\omega_{1}, \omega_{2}\right) A_{1} A_{2} e^{i\left(\omega_{1}+\omega_{2}\right) t} \\
& +H_{2}^{(j)}\left(\omega_{2}, \omega_{2}\right) A_{2}^{2} e^{i 2 \omega_{2} t}
\end{aligned}
$$

where $H_{1}^{(j)}$ and $H_{2}^{(j)}$ represent the first and second order frequency-domain Volterra kernels for the output component $W_{j}$. Substituting this expression in the ODE describing the system these kernels can be obtained. For examples on mechanical systems see Worden et al. ${ }^{23}$ and for aeroelastic systems with structural nonlinearities see Marzocca et al. ${ }^{21}$

\section{III.A. Linearized-frequency domain method for a second order functional series expansion}

Now the HP method is applied to the semi-discrete form (also known as method of lines) of a PDE. Several authors have obtained Volterra kernels for some particular nonlinear PDEs prior to an spatial discretization. These kernels are the solution to a set of ODEs which depends on the particular nonlinear PDE under consideration, see for example Hélie et al ${ }^{27}$ for an application to the viscous Burgers' equation. The novelty of the approach presented in this work consists in the application of a spatial discretization of the PDE by any of the available methods (finite differences, finite volume or finite elements) first, the application of a second order Taylor's expansion next and finally the implementation of the HP method to obtain a general form for the first and second order Volterra kernels, leading to the LFD2 method. Thus, unlike the methods for the determination of the Volterra kernels for some particular PDEs where a set of ODEs is obtained, due to the spatial discretization and the Taylor's expansion two complex linear algebraic systems of equations are obtained instead.

A single-input system of $N$ degrees of freedom in semi-discrete form is described by:

$$
\frac{d \hat{\mathbf{W}}}{d t}=\mathbf{R}(\hat{\mathbf{W}}, \hat{u})
$$

where $\hat{\mathbf{W}}(t) \in \mathbb{R}^{N}, \hat{u} \in \mathbb{R}$ is the input and $\mathbf{R}(\hat{\mathbf{W}}, \hat{u}) \in \mathbb{R}^{N}$ is the residual function. Note that no explicit dependency on the position has been considered after the spatial discretization as the grid does not undergo any deformation for the systems considered in this work. For unsteady flow phenomena is interesting to split the variables into a time-independent steady and an incremental unsteady parts, $\hat{\mathbf{W}}(t)=\mathbf{W}_{0}+\mathbf{W}(t)$ and $\hat{u}(t)=u_{0}+u(t)$. In the steady-state the time derivative is zero and thus the derivative for the incremental variables is:

$$
\frac{d \mathbf{W}}{d t}=\mathbf{R}\left(\mathbf{W}_{0}+\mathbf{W}, u_{0}+u\right)
$$

Applying now a Taylor's expansion to the vector-valued residual function around the steady state $\mathbf{W}_{0}$ reached by the input $u_{0}$ :

$$
\mathbf{R}\left(\mathbf{W}_{0}+\mathbf{W}, u_{0}+u\right) \approx \mathbf{R}\left(\mathbf{W}_{0}, u_{0}\right)+\mathbf{J}_{w}\left(\mathbf{W}_{0}, u_{0}\right) \mathbf{W}+\mathbf{J}_{u}\left(\mathbf{W}_{0}, u_{0}\right) u+\frac{1}{2} \mathbf{Q}\left(\mathbf{W}_{0}, u_{0}\right)\left(\left[\mathbf{W}^{T} u\right]^{T}\right)^{\otimes 2},
$$

where $\mathbf{J}_{w}\left(\mathbf{W}_{0}, u_{0}\right)=\frac{\partial \mathbf{R}}{\partial \mathbf{W}^{T}}\left(\mathbf{W}_{0}, u_{0}\right)$ and $\mathbf{J}_{u}\left(\mathbf{W}_{0}, u_{0}\right)=\frac{\partial \mathbf{R}}{\partial u}\left(\mathbf{W}_{0}, u_{0}\right)$ are the Jacobian matrices, $\otimes$ represents the Kronecker product ${ }^{28}$ and the power $\otimes 2$ refers again to the Kronecker product:

$$
\left(\left[\mathbf{W}^{T} u\right]^{T}\right)^{\otimes 2}=\left[\mathbf{W}^{T} u\right]^{T} \otimes\left[\mathbf{W}^{T} u\right]^{T} .
$$

The matrix $\mathbf{Q}\left(\mathbf{W}_{0}, u_{0}\right)$ contains the terms of the Hessian tensor reordered in a rectangular form (note that the symbol $\mathbf{Q}$ is used here for the Hessian in order to avoid confusion with the Volterra kernels in the frequency domain) as: $\stackrel{29}{\underline{29}}$ 


$$
\mathbf{Q}\left(\mathbf{W}_{0}, u_{0}\right)=\left[\begin{array}{c}
Q_{1}\left(\mathbf{W}_{0}, u_{0}\right) \\
\vdots \\
Q_{j}\left(\mathbf{W}_{0}, u_{0}\right) \\
\vdots \\
Q_{N}\left(\mathbf{W}_{0}, u_{0}\right)
\end{array}\right]=\left[\begin{array}{c}
\operatorname{vec}\left(\frac{\partial}{\partial\left(\left[\mathbf{W}^{T} u\right]\right)}\left[\left(\frac{\partial R_{1}}{\partial\left(\left[\mathbf{W}^{T} u\right]\right)}\left(\mathbf{W}_{0}, u_{0}\right)\right)^{T}\right]\right)^{T} \\
\vdots \\
\operatorname{vec}\left(\frac{\partial}{\partial\left(\left[\mathbf{W}^{T} u\right]\right)}\left[\left(\frac{\partial R_{j}}{\partial\left(\left[\mathbf{W}^{T} u\right]\right)}\left(\mathbf{W}_{0}, u_{0}\right)\right)^{T}\right]\right)^{T} \\
\vdots \\
\operatorname{vec}\left(\frac{\partial}{\partial\left(\left[\mathbf{W}^{T} u\right]\right)}\left[\left(\frac{\partial R_{N}}{\partial\left(\left[\mathbf{W}^{T} u\right]\right)}\left(\mathbf{W}_{0}, u_{0}\right)\right)^{T}\right]\right)^{T}
\end{array}\right],
$$

where each row $Q_{j}\left(\mathbf{W}_{0}, u_{0}\right)$ corresponds to the Hessian matrix of the component $j$. Alternatively, the matrix $\mathbf{Q}\left(\mathbf{W}_{0}, u_{0}\right) \in \mathbb{R}^{N \times(N+1)^{2}}$ may be regarded as the 1-mode matricization of the Hessian tensor of third order

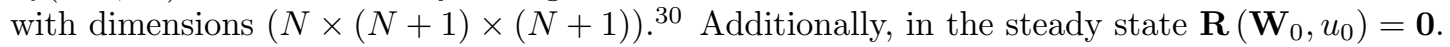

For the determination of the first order kernel in the frequency domain, which corresponds to the transfer function matrix of the linearized system, the single-tone input $u(t)=A_{1} e^{i \omega t}$ is imposed. Substituting the output $W_{j}=H_{1}^{(j)}(\omega) A_{1} e^{i \omega t}$ into Eq. 7 gives:

$$
\left(i \omega \mathbf{I}-\mathbf{J}_{w}\left(\mathbf{W}_{0}, u_{0}\right)\right) \mathbf{H}_{1}(\omega)=\mathbf{J}_{u}\left(\mathbf{W}_{0}, u_{0}\right),
$$

which is a complex linear system of equations for the determination of $\mathbf{H}_{1}(\omega)=\left[H_{1}^{(1)}(\omega) \cdots H_{1}^{(j)}(\omega) \cdots H_{1}^{(N)}(\omega)\right]^{T}$. Eq. 8 is equivalent to the solution in the frequency domain of the linearized system ${ }^{2}$ (in the current work the grid deformation has not been taken into account). Dropping the explicit dependency on $\left(\mathbf{W}_{0}, u_{0}\right)$ for the sake of clarity Eq. 8 reduces to the following equation for the component $j$ :

$$
i \omega H_{1}^{(j)}(\omega)-\sum_{k=1}^{N} \frac{\partial R_{j}}{\partial W_{k}} H_{1}^{(k)}(\omega)=\frac{\partial R_{j}}{\partial u}
$$

For the second order kernel the two-tone input signal of Eq. 5 is imposed and the relation given by Eq. 6 is substituted in Eq. 7. After some algebraic manipulations and equating the terms multiplying $A_{1} A_{2} e^{i\left(\omega_{1}+\omega_{2}\right) t}$ the following relation is obtained:

$$
\left(i\left(\omega_{1}+\omega_{2}\right) \mathbf{I}-\mathbf{J}_{w}\left(\mathbf{W}_{0}, u_{0}\right)\right) \mathbf{H}_{2}\left(\omega_{1}, \omega_{2}\right)=\frac{1}{2} \mathbf{Q}\left(\mathbf{W}_{0}, u_{0}\right)\left(\left[\begin{array}{lll}
\mathbf{H}_{1}^{T}\left(\omega_{1}\right) & 1
\end{array}\right]^{T} \otimes\left[\mathbf{H}_{1}^{T}\left(\omega_{2}\right) 1\right]^{T}\right),
$$

where $\mathbf{H}_{2}\left(\omega_{1}, \omega_{2}\right)=\left[H_{2}^{(1)}\left(\omega_{1}, \omega_{2}\right) \cdots H_{2}^{(j)}\left(\omega_{1}, \omega_{2}\right) \cdots H_{2}^{(N)}\left(\omega_{1}, \omega_{2}\right)\right]^{T}$ and the symmetry of the second order cross derivatives has been taken into account. Again, Eq. 9 represents a complex linear system of equations for each frequency pair value $\left(\omega_{1}, \omega_{2}\right)$ and is the key component of the LFD2 method developed in this work. Eq. 9 reduces to the following relation for the component $j$ :

$$
\begin{aligned}
& i\left(\omega_{1}+\omega_{2}\right) H_{2}^{(j)}\left(\omega_{1}, \omega_{2}\right)-\sum_{k=1}^{N} \frac{\partial R_{j}}{\partial W_{k}} H_{2}^{(k)}\left(\omega_{1}, \omega_{2}\right) \\
= & \frac{1}{2}\left(\sum_{k=1}^{N} \sum_{l=1}^{N} \frac{\partial^{2} R_{j}}{\partial W_{k} \partial W_{l}} H_{1}^{(k)}\left(\omega_{1}\right) H_{1}^{(l)}\left(\omega_{2}\right)+\frac{\partial^{2} R_{j}}{\partial u^{2}}+\sum_{k=1}^{N} \frac{\partial^{2} R_{j}}{\partial W_{k} \partial u}\left(\sum_{l=1}^{N} H_{1}^{(k)}\left(\omega_{l}\right)\right)\right)
\end{aligned}
$$

Note the similarity of Eq. 9 with the Volterra kernels obtained by several authors $\$ 31$ for bilinear systems in the input (that is, containing solely linear terms with respect to the input $u(t)$ ).

Both the Jacobian $\mathbf{J}_{w}\left(\mathbf{W}_{0}, u_{0}\right)$ and Hessian $\mathbf{Q}\left(\mathbf{W}_{0}, u_{0}\right)$ matrices appear in Eq. 9 and thus they are required for the solution of the second order Volterra kernel. Note that the solution of the linear system given by Eq. 9 requires the knowledge of the first order Volterra kernel, which is obtained by solving Eq. 8 first. The linear algebraic systems specified by Eqs. 8 and 9 are solved for the frequencies interval $-\omega_{\max } \leq \omega_{1} \leq \omega_{\max }$ for the first order kernel and $-\omega_{\max } \leq \omega_{1} \leq \omega_{\max }$ together with $-\omega_{\max } \leq \omega_{2} \leq \omega_{\max }$ 
for the second order kernel, where the maximum circular frequency $\omega_{\max }$ is beyond the frequencies of interest for the particular phenomena under consideration. The range of frequencies at which the systems given by Eqs. 8 and 9 need to be solved may however be reduced, as some properties of the Volterra kernels in the frequency domain can be used: 34

$$
\begin{gathered}
H_{1}^{(j)}\left(-\omega_{1}\right)=\bar{H}_{1}^{(j)}\left(\omega_{1}\right), \\
H_{2}^{(j)}\left(\omega_{1}, \omega_{2}\right)=H_{2}^{(j)}\left(\omega_{2}, \omega_{1}\right), \\
H_{2}^{(j)}\left(-\omega_{1},-\omega_{2}\right)=\bar{H}_{2}^{(j)}\left(\omega_{1}, \omega_{2}\right),
\end{gathered}
$$

where $\bar{H}_{1}^{(j)}$ and $\bar{H}_{2}^{(j)}$ denote the complex conjugates. According to Eq. 10 , only the region $0 \leq \omega_{1} \leq \omega_{\max }$ needs to be computed by Eq. 8 for the first order kernel. For the second order kernel, only the region $-\omega_{\max } \leq \omega_{1} \leq \omega_{\max }$ and $\left|\omega_{1}\right| \leq \omega_{2} \leq \omega_{\max }$ needs to be considered, as the values of the second order Volterra kernel outside this region are known by considering Eqs. 11 and 12. In Fig. 1 the region ABC where Eq. 9 needs to be solved for the second order Volterra kernel is highlighted. For illustration purposes two points within this region $\left(\omega_{a}, \omega_{b}\right)$ and $\left(\omega_{c}, \omega_{d}\right)$ are shown (note that these two points may be randomly chosen within the highlighted region, but have been shown somewhat symmetrical with respect to the $\omega_{2}$ axis for the sake of clarity). From these two values, the indicated values outside the highlighted region ABC can be deduced. Thus, considering the complete domain given by the highlighted region the complete plane $\left(\omega_{1}, \omega_{2}\right)$ is covered. For generality the maximum frequency $\omega_{\max }$ is not shown in Fig. 1 as this discussion still holds if an infinity range of frequency values were under consideration.

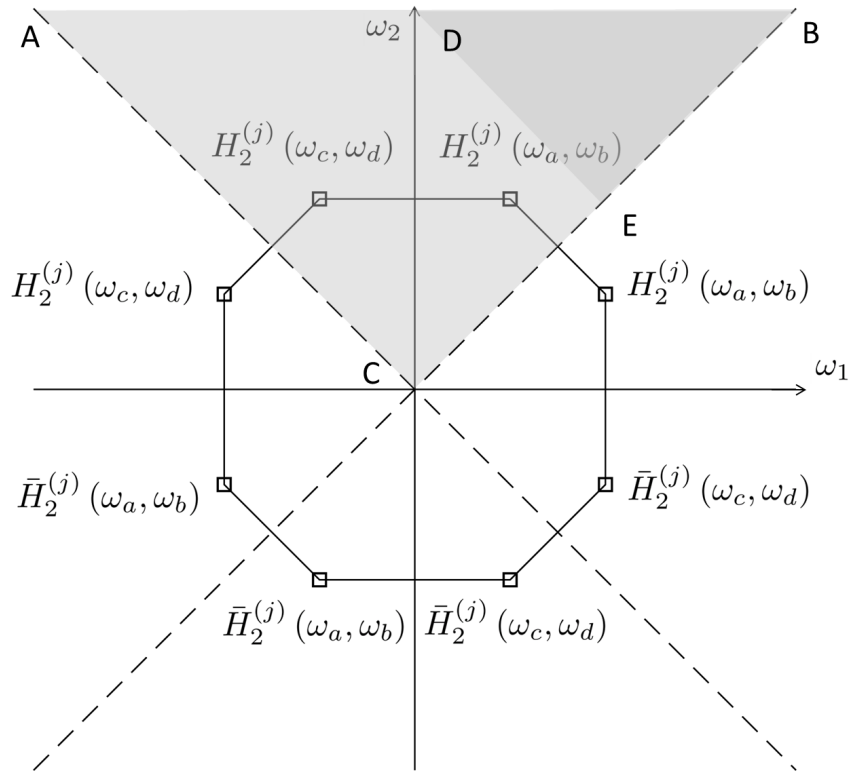

Figure 1. Region of computation for the second order Volterra kernel in the frequency domain $H_{2}^{(j)}\left(\omega_{1}, \omega_{2}\right)$.

By choosing an odd (in order to include the frequency zero) number $N_{f}$ of sample frequencies and considering the properties given by Eqs. 10 to 12 , the linear system of Eq. 8 needs to be solved for a number of frequencies equal to $\left(N_{f}+1\right) / 2$ for the first order Volterra kernel. The linear system of Eq. 9 for the second order Volterra kernel needs to be solved for a number $\left(N_{f}+1\right)^{2} / 4$ of sample frequencies. Thus the total number of linear systems to be solved for the generation of the nonlinear ROM for a second order functional series expansion of a single-input nonlinear PDE is $(1 / 2)\left[\left(N_{f}+1\right)+\left(N_{f}+1\right)^{2} / 2\right]$.

For the solution of the complex linear systems given by Eqs. 8 and 9 a different number of techniques such as the preconditioned Krylov generalized minimal residual (GMRES) 2$]$ can be used. Also an iteration in 
an additional pseudotime variable until an steady solution is reached, as typically done in the HB technique, 9 may be used. The aim of this work is to establish the general solution for the first and second order kernels of nonlinear PDEs by means of the solution to the two linear algebraic systems given by Eqs. 8 and 9 and thus the influence of different linear solvers has not been further investigated. Note that if the inverse matrix in Eq. 8 is available for the computation of the second order Volterra kernel, the inverse matrix required for the determination of the second order Volterra kernel by Eq. 9 must be computed solely for the upper right region BDE highlighted in Fig. 1 by setting $\omega=\omega_{1}+\omega_{2}$, additionally reducing by a factor of 4 the number of inverse matrices to be computed.

For the applications of Section IV] a banded linear solver which uses LU decomposition with partial pivoting has been chosen, as the maximum number unknowns is lower than $N=10^{3}$.

Unlike other techniques such as the perturbation and Adomian methods or the more general homotopy analysis method ${ }^{24}$ the solution of the higher order contributions due to the nonlinearities does not depend on the input $u$, as it does not appear in neither of the Eqs. 8 and 9 . This is of particular interest in the aeroelasticity field, as both the structural and flow equations must be coupled to solve for the physical phenomena. The coupling of a weak nonlinear system describing the unsteady flow with a linear system describing the structural behaviour would give rise in this setting to an integral equation of the first kind in the frequency domain for the dynamic response which can be efficiently solved as the nonlinear PDE describing the flow does not have to be numerically integrated in time. For the time-domain representation an equivalent integro-differential equation due to the convolution integrals of Eq. 3 is obtained. However, a second order functional series expansion can also be represented in the time domain by a bilinear state-space form,$\stackrel{35}{,}$ which would then replace the integro-differential formulation by a set of nonlinear ODEs. None of these possibilities are the aim of this work and are proposed for future work.

\section{III.B. Solution}

Once the Volterra kernels in the frequency domain have been computed the output $\mathbf{W}$ can be obtained using Eq. 4 for the incremental variables. An inverse Fourier transform for each component $j$ provides the signal in the time domain $W_{j}(t)$. A frequency-discrete version of the process described by Lang et al $\frac{36}{6}$ has been implemented for the integral in the second term in Eq. 4. This term produces frequencies in the interval $\left(-2 \omega_{\max }, 2 \omega_{\max }\right)^{36}$ but the frequencies beyond $\left|\omega_{\max }\right|$ have been neglected under the assumption that the nonlinearities are concentrated in the frequency range of interest. If the input signal is not absolutely

integrable, that is, the integral $\int_{0}^{\infty}|u(\tau)| d \tau$ is not bounded, the time domain version given by Eq. 3 is used for the computation of the output component $W_{j}(t)$. In this case a bidimensional convolution is involved. The total value of the variable $\hat{W}_{j}(t)$ is recovered by adding the steady-state value, $\hat{W}_{j}(t)=W_{0}+W_{j}(t)$.

In the case of a single-tone harmonic input $u(t)=A_{1} e^{i \omega_{0} t}$ at frequency $\omega_{0}$ and if the transient of the signal $\mathbf{W}(t)$ is of no interest, a simplified relation for the component $W_{j}(t)$ in the time domain can be used! 26

$$
\begin{aligned}
W_{j}(t)= & \frac{A_{1}}{2} H_{1}^{(j)}\left(\omega_{0}\right) e^{i \omega_{0} t}+\frac{\bar{A}_{1}}{2} H_{1}^{(j)}\left(\omega_{0}\right) e^{-i \omega_{0} t} \\
& +\frac{A_{1}^{2}}{4} H_{2}^{(j)}\left(\omega_{0}, \omega_{0}\right) e^{i 2 \omega_{0} t}+\frac{\bar{A}_{1}^{2}}{4} H_{2}^{(j)}\left(-\omega_{0},-\omega_{0}\right) e^{-i 2 \omega_{0} t}+\frac{\left|A_{1}\right|^{2}}{2} H_{2}^{(j)}\left(-\omega_{0}, \omega_{0}\right) .
\end{aligned}
$$

For both Eqs. 3 and 13 the complete output frequency interval $\left(-2 \omega_{\max }, 2 \omega_{\max }\right)$ is taken into account.

The topic of the convergence of the Volterra or functional series expansion is very challenging and so far there is no general method which can be used to determine its interval of convergence. ${ }^{16}$ Certainly this topic has to be addressed in future work.

\section{Application cases}

In this section the LFD2 method and the resulting nonlinear ROM as described in Section III is applied to two different systems. The aim here is to show the validity of Eqs. 8 and 9 for the determination of the Volterra kernels of first and second order in the frequency domain independent of the spatial discretization applied to the PDE. The first system, described by the Burgers' viscous equation, has been considered by Silva ${ }^{18}$ for the identification of the first and second order kernels after spatial discretization by the finite differences method. 
As stated by Guo et al ${ }^{37}$ many theoretical and numerical studies are tested on the Burgers' equation prior to their application to more complex PDEs. The Burgers' equation can be considered as a simpler model compared to the Navier-Stokes equations for modelling flow phenomena due to the presence of the nonlinear convection term, which makes the analysis of the equation in the frequency domain very challenging.

Hélie et al ${ }^{27}$ have computed the Volterra kernels of a Burgers' acoustic model with a fractional derivative by using block-diagram representations of the nonlinear system. The kernels depend additionally on the spatial variable and are obtained by solving a sequence of linear ODEs. Guo et al ${ }^{37}$ computed the spatially dependent Volterra kernels of the nonlinear viscous Burgers' equation in the frequency domain and considered steady-state responses to harmonic inputs. Battaglia et al ${ }^{38}$ applied the Volterra series to a nonlinear heat diffusion problem, obtaining a sequence of linear ODEs for the multidimensional Laplace transforms of the Volterra kernels. In these three cases the analysis is based on the spatial continuous version of the Burgers' equation and is only valid for the particular PDE under consideration. In this work the equations derived in Section III.A are valid for general PDE in semi-discrete form after spatial discretization.

The second system involves the quasi-one dimensional nonlinear Euler equations describing the unsteady flow in a nozzle with variable cross section in conservative form. Here the finite volume method is chosen, showing the applicability of the method presented in Section III independent of the scheme used for the spatial discretization. The boundary conditions are formulated in characteristic form $\frac{39}{}$

The computation of the Volterra kernels in the frequency domain by means of Eqs. 8 and 9 requires the determination of the Jacobian and Hessian operators of the residual function in semi-discrete form. For applications involving only the first order Volterra kernel, which is equivalent to the linearized system in the incremental variables, an analytical expression of the Jacobian matrix may be used, see for instance Dwight. 40 Additionally to the Jacobian matrix the Hessian matrix is required in this work, see Eq. 9. In order to avoid the analytical derivation of the second order derivatives they have been obtained by means of the automatic differentiation (AD) technique 4142$]$ Unlike the finite difference method which is commonly used in combination with the analytical Jacobian to obtain the first order derivatives, ${ }^{[2]}$ the $\mathrm{AD}$ technique does not suffer of round-off errors.

\section{IV.A. Burgers equation}

In this section the viscous Burgers' equation for the single output variable $\hat{W}(t)$ with a nonlinear convective term is considered:

$$
\frac{\partial \hat{W}}{\partial t}+\hat{W} \frac{\partial \hat{W}}{\partial x}=v \frac{\partial^{2} \hat{W}}{\partial x^{2}}
$$

where $-1 \leq x \leq 1, t \geq 0$ and the viscosity has been set to $\nu=0.01$. All variables are nondimensional. The input is specified by the boundary condition $\hat{u}(t)=\hat{W}(t, x=-1)$. The problem of interest is to predict the nonlinear incremental unsteady velocity $W(x, t)$ around a steady value, $W(x, t)=\hat{W}(x, t)-W_{0}(x)$.

For the spatial discretization a total number of $N=81$ nodes has been considered together with a second order central finite differences scheme. As a reference solution the direct numerical integration in the time variable of Eq. 14 by an explicit Runge-Kutta method of fifth order with a time step of 0.005 has been chosen. First the steady values $W_{0 j}(j=1, \ldots, N)$ corresponding to a steady input $u_{0}=0.1$ are considered as an equilibrium condition. For the incremental variables the incremental input is $u(t)=\hat{u}(t)-u_{0}$.

For the computation of the Volterra kernels in the frequency domain different frequency discretization sets have been used for the solution of Eqs. 8 and 9 . For the first order kernel a maximum circular frequency of $\omega_{\max }=200 \pi$ with a regular spacing of $\Delta \omega=0.004 \pi$ has been chosen. This low frequency spacing takes care of the long time required for the first order time-domain Volterra kernel to damp out due to the small value of the viscosity constant $v=0.01$. For the second order kernel a maximum circular frequency of $\omega_{\max }=5 \pi$ with a regular spacing of $\Delta \omega=0.067 \pi$ has been used. For the solution of Eq. 9 the first order Volterra kernels have been interpolated to the second order frequency dataset. The possibility of choosing different frequency datasets for the different orders of the Volterra kernels offers a high flexibility in the present method.

Now the response to a 1 -cosine pulse input as specified in Eq. 15 with $u_{1}=0.07$ and $t_{1}=0.5$ is considered: 


$$
u(t)= \begin{cases}\frac{u_{1}}{2}\left[1-\cos \left(2 \pi t / t_{1}\right)\right], & 0 \leq t \leq t_{1}, \\ 0, & t>t_{1} .\end{cases}
$$

Fig. 2 shows a comparison of the output $\hat{W}_{j}(t)=W_{0 j}+W_{j}(t)$ between the linearized solution containing only the first order Volterra kernel (LFD), the nonlinear ROM including the first and second order Volterra kernels (LFD2) and the reference results obtained by time integration of Eq. 14 (labeled as PDE) for the component $j$ corresponding to the position $x=-0.5$. In this case the incremental output $W_{j}$ has been computed using Eq. 4. The steady-state response to a harmonic single-tone input of period $T=5$ and maximum amplitude of $|u(t)|=0.08$ is considered in Fig. 3. where the incremental output $W_{j}$ has been obtained by Eq. 13 in this case. It is clear from Figs. 2 and 3 that the nonlinear ROM corresponding to the second order functional series expansion (LFD2) is able to properly capture the (weak) nonlinearities produced by the nonlinear convective term for arbitrary input signals.

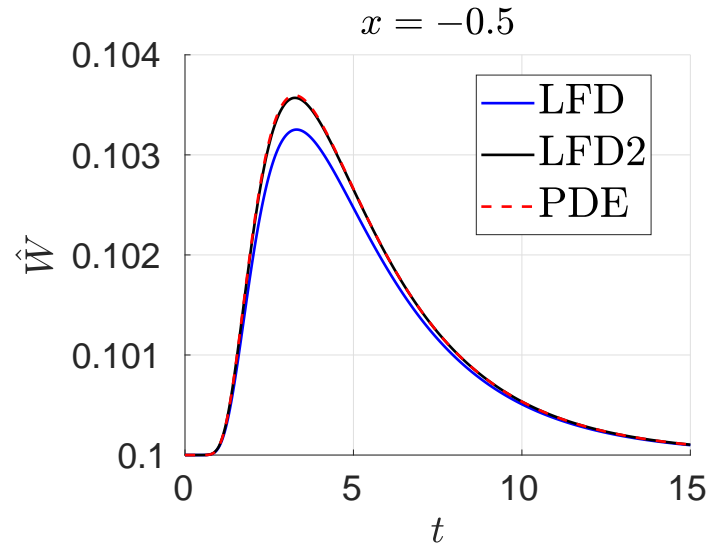

Figure 2. Response at $x=-0.5$ to a 1-cosine pulse input.

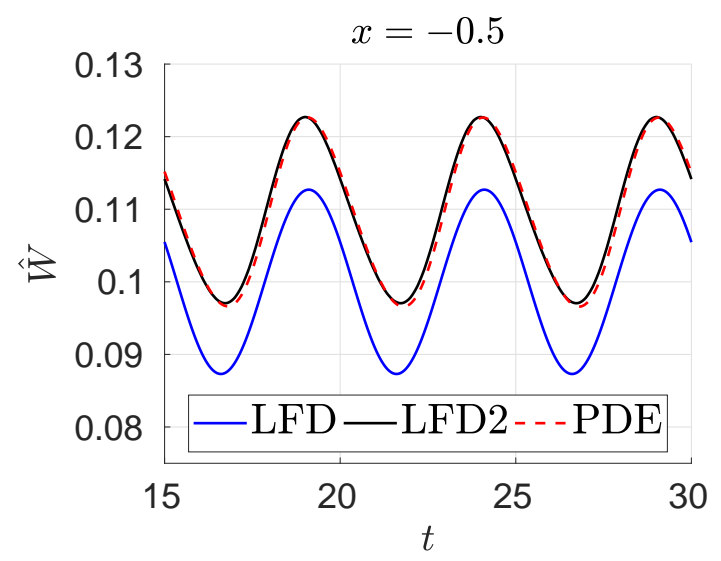

Figure 3. Response at $x=-0.5$ to a harmonic input.

\section{IV.B. Quasi one-dimensional Euler equations}

In this section the quasi-one dimensional unsteady compressible flow over a nozzle of variable area $A(x)$ and described by the Euler equations is considered. The quasi-one dimensional Euler equations in conservative and differential form are:

$$
A(x) \frac{\partial \hat{\mathbf{W}}}{\partial t}+\frac{\partial(\mathbf{F}(\hat{\mathbf{W}}) A(x))}{\partial x}=\mathbf{S}(\hat{p}) \frac{d A(x)}{d x},
$$

where $\hat{\mathbf{W}}$ represents the vector of conservative variables, $\mathbf{F}$ the vector of convective fluxes and $\mathbf{S}$ the source term:

$$
\hat{\mathbf{W}}=\left[\begin{array}{c}
\hat{\rho} \\
\hat{\rho} \hat{u} \\
\hat{\rho} \hat{E}
\end{array}\right], \mathbf{F}(\hat{\mathbf{W}})=\left[\begin{array}{c}
\hat{\rho} \hat{u} \\
\hat{p}+\hat{\rho} \hat{u}^{2} \\
\hat{\rho} \hat{H} \hat{u}
\end{array}\right], \mathbf{S}(\hat{p})=\left[\begin{array}{c}
0 \\
\hat{p} \\
0
\end{array}\right]
$$

with the flow variables $\hat{\rho}, \hat{u}$ and $\hat{p}$ representing the density, velocity and pressure respectively. Additionally the following relations for the total enthalpy $\hat{H}$ and for the pressure $\hat{p}$ by means of the state equation are considered:

$$
\hat{H}=\hat{E}+\frac{\hat{p}}{\hat{\rho}}, \hat{p}=(\gamma-1) \hat{\rho}\left(\hat{E}-\frac{\hat{u}^{2}}{2}\right)
$$


where $\hat{E}$ is the total energy. The area distribution $A(x)$ as proposed by Blazek ${ }^{43}$ has been used:

$$
\begin{gathered}
A(x)=1+\frac{\left(A_{\text {in }}-1\right)}{2}\left(1+\cos \left(\frac{x \pi}{x_{t}}\right)\right), 0 \leq x \leq x_{t}, \\
A(x)=1+\frac{\left(A_{\text {out }}-1\right)}{2}\left(1-\cos \left(\frac{\left(x-x_{t}\right) \pi}{1-x_{t}}\right)\right), x_{t} \leq x \leq 1,
\end{gathered}
$$

where the inlet and outlet areas have been set to $A_{\text {in }}=1.5\left(\mathrm{~m}^{2}\right)$ and $A_{\text {out }}=2.5\left(\mathrm{~m}^{2}\right)$ respectively. The throat is located at $x_{t}=0.375(\mathrm{~m})$. The input is defined as the pressure at the outlet position, $\hat{u}(t)=\hat{p}(t, x=1)=\hat{p}_{\text {out }}(t)$.

The spatial discretization corresponds to a finite volume scheme with a dual cell methodology and a total number of 129 internal nodes, corresponding to a total of $N=387$ degrees of freedom. Scalar artificial dissipation according to the JST scheme ${ }^{44}$ with constants $k_{2}=1 / 2$ and $k_{4}=1 / 64$ has been added. External boundaries have been handled with ghost cells and the flow variables at these ghost cells have been determined using characteristic boundary conditions ${ }^{39}$ After application of the finite volume scheme the semi-discrete form of the quasi-one dimensional Euler equations is then given by:

$$
\frac{d \hat{\mathbf{W}}}{d t}=\mathbf{R}\left(\hat{\mathbf{W}}, \hat{p}_{\text {out }}\right),
$$

with the residual including the convective and the artificial viscosity fluxes together with the source term. An explicit Runge-Kutta multistage scheme of fifth order is used for the time integration of Eq. 16. For the steady solution local time stepping together with an implicit residual smoothing technique are used for convergence acceleration.

The maximum circular frequency has been set to $\omega_{\max }=8.976 \cdot 10^{3}(\mathrm{rad} / \mathrm{s})$ and the spacing is $\triangle \omega=25.15$ $(\mathrm{rad} / \mathrm{s})$ for the computation of both first and second order Volterra kernels.

A steady-state condition defined by a total pressure of $10^{5}(\mathrm{~Pa})$ and a total temperature of $288(\mathrm{~K})$ at the inlet and a static pressure at the outlet of $9.8 \cdot 10^{4}(\mathrm{~Pa})$ is considered, see Fig. 8 resulting in a subsonic flow all over the nozzle. Thus, the divergent part acts in this case as a diffuser. Additionally the analytical solution is compared with the numerical solution of the Euler equaions (PDE). The proposed method of Section III is now applied to the incremental conservative variables $\mathbf{W}$.

Once the steady-state condition has been defined, the corresponding first and second order Volterra kernels are computed in the frequency domain by means of Eqs. 8 and 9 Fig. 4 shows the first order frequency-domain Volterra kernel corresponding to the incremental pressure $\hat{p}_{j}(t)$ at a position $x=0.40$ $(\mathrm{m})$. By means of a Fourier transform the first order Volterra kernel is converted into the time domain, see Fig. 5 .

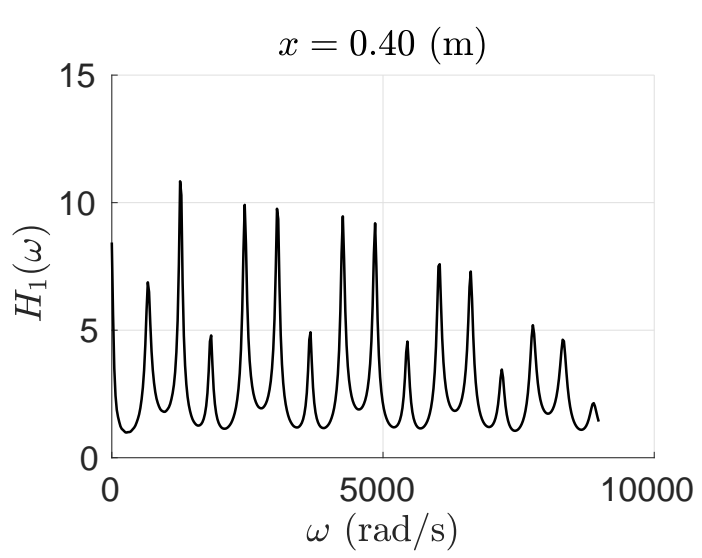

Figure 4. First order Volterra kernel corresponding to the pressure $\hat{p}_{j}(t)$ at position $x=0.40(\mathbf{m})$. Frequency domain.

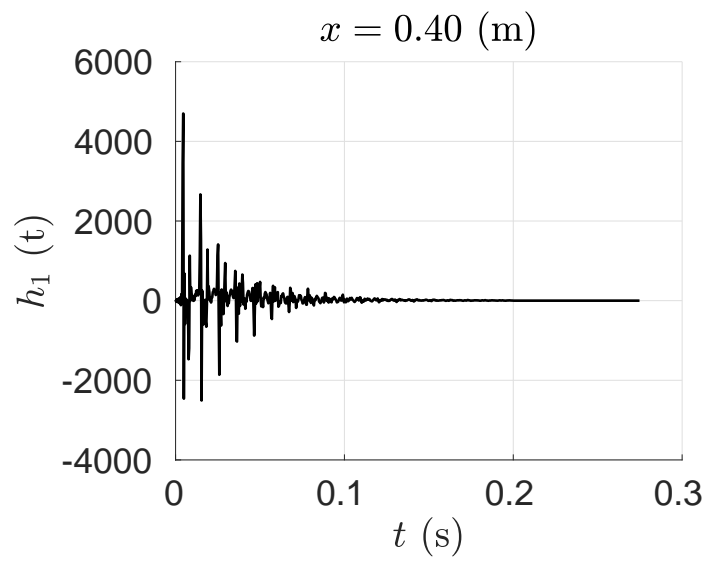

Figure 5. First order Volterra kernel corresponding to the pressure $\hat{p}_{j}(t)$ at position $x=0.40(\mathbf{m})$. Time domain. 
Similarly, Figs. 6 and 7 show the second order Volterra kernels in the frequency and time domains respectively for the incremental pressure at the same position. The second order Volterra kernel has been obtained by solving Eq. 9 in the frequency domain and the corresponding time-domain counterpart has been obtained by means of an inverse bidimensional Fourier transform.

$$
x=0.40(\mathrm{~m})
$$

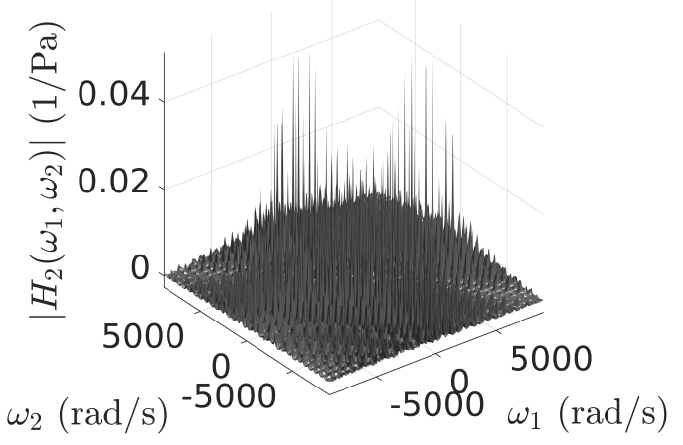

Figure 6. Second order Volterra kernel corresponding to the pressure $\hat{p}_{j}(t)$ at position $x=0.40(\mathbf{m})$. Frequency domain.

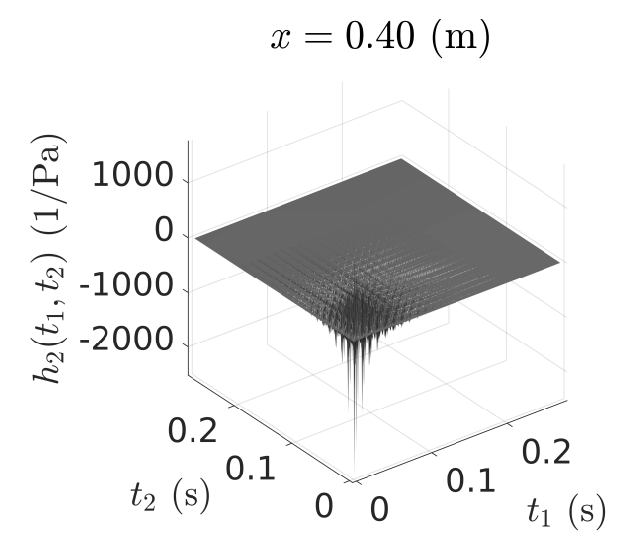

Figure 7. Second order Volterra kernel corresponding to the pressure $\hat{p}_{j}(t)$ at position $x=0.40(\mathbf{m})$. Time domain.

Fig. 9 shows the static pressure $\hat{p}_{j}(t)$ at a position just behind the throat, $x=0.40(\mathrm{~m})$. The input is a 1-cosine pulse perturbation on the back static pressure as defined by Eq. 15 with $u_{1}=2352(\mathrm{~Pa})$ and $t_{1}=0.025(\mathrm{~s})$.

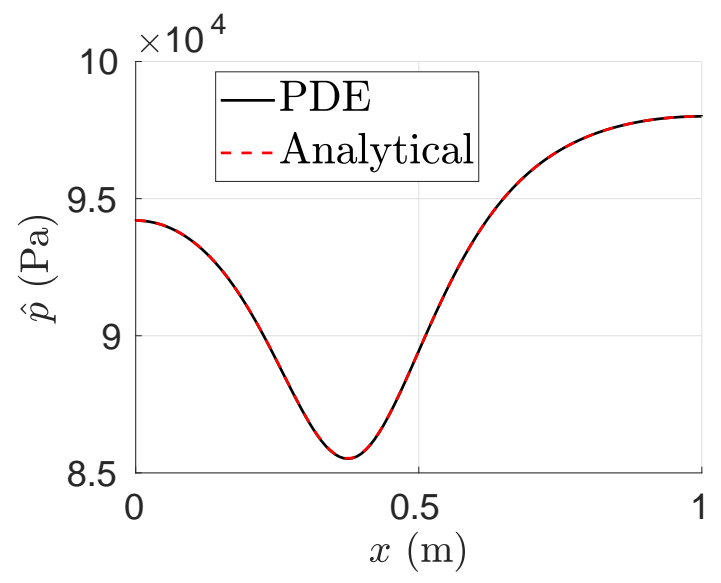

Figure 8. Steady pressure distribution (subsonic compressible case).

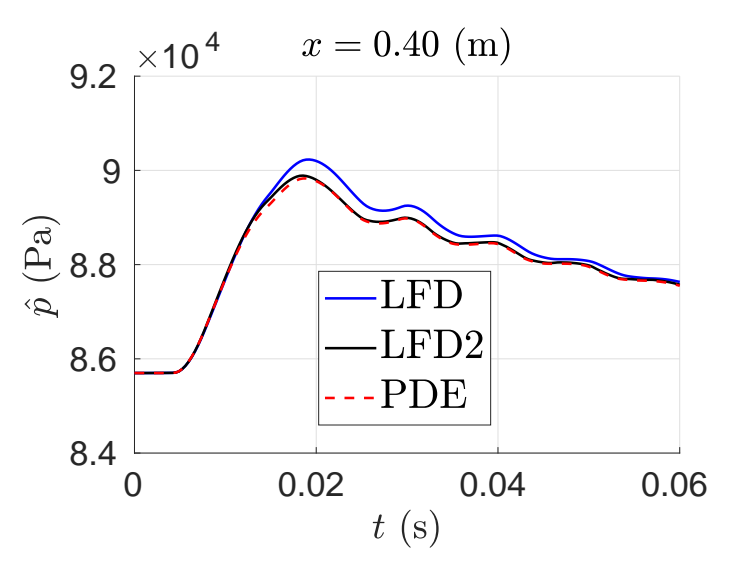

Figure 9. Pressure $\hat{p}_{j}(t)$ at position $x=0.40(\mathbf{m})$ for a 1-cosine back pressure perturbation.

Fig. 10 shows the pressure $\hat{p}_{j}(t)$ for a quasi-random back pressure perturbation of maximum amplitude 2352 (Pa) whereas in Fig. 11 the steady-state regime due to a harmonic back pressure perturbation with frequency $\omega_{0}=83.79(\mathrm{rad} / \mathrm{s})$ and an amplitude of $4704(\mathrm{~Pa})$ has been considered. The nonlinear ROM including a second order functional series expansion (LFD2) is thus able to predict weak nonlinearities of the system described by the PDE for arbitrary inputs. In all cases shown in Figs. 911 the solution labeled as LFD includes only the first order Volterra kernel and thus represents the linearized solution.

Regarding the computational effort, there is a first offline phase which is required in order to build the ROM where the Volterra kernels and a subsequent online phase which makes use of these kernels to compute the (weak) nonlinear response. Thus the suitability of the presented nonlinear ROM depends on the number of computations with different inputs which needs to be considered. For a high number of excitations it is very efficient and very flexible, as explicit formulas for absolutely integrable, non integrable, and multi-tone 


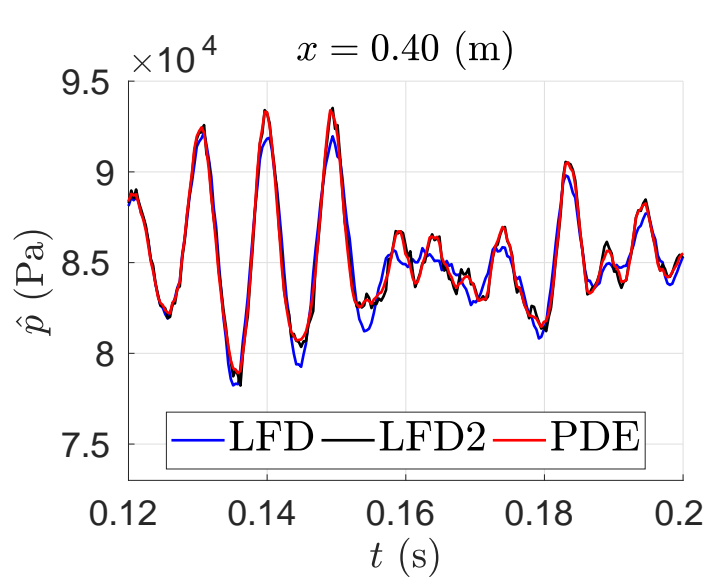

Figure 10. Pressure $\hat{p}_{j}(t)$ at position $x=0.40(\mathbf{m})$ for a quasi-random back pressure perturbation.

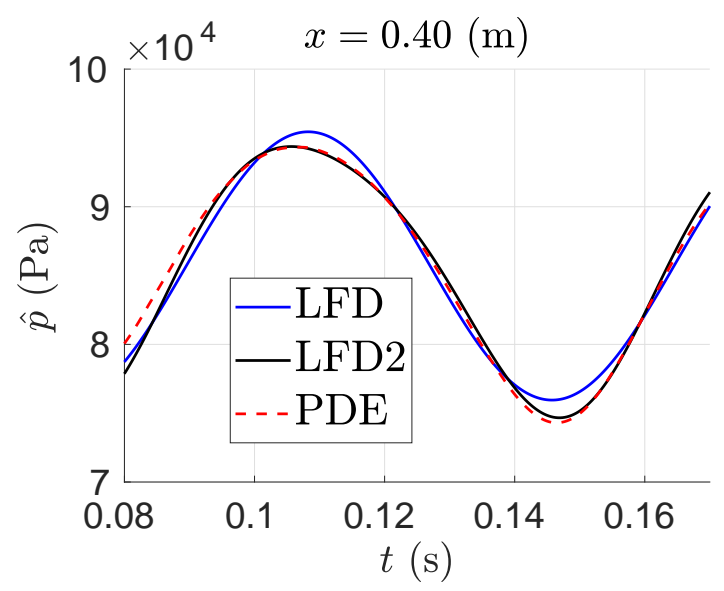

Figure 11. Pressure $\hat{p}_{j}(t)$ at position $x=0.40(\mathbf{m})$ for a harmonic back pressure perturbation.

signals are available for the particular case of interest, see Eqs. 4, 3, and 13 respectively. Any of these implementations can save up to several orders of magnitude in computational time even for one input signal in the case where many degrees of freedom are involved, at the expense of increasing the computational cost of the previous offline phase for the determination of the Volterra kernels.

A main advantage of the present method is that as for the linearized PDE solvers, the computational cost for obtaining the incremental output response $\mathbf{W}$ is the same independent of the size of the problem once the Volterra kernels have been obtained. Additionally, once the Volterra kernels are known a small subset of interest within the output vector $\mathbf{W}$ can be computed. In order to further evaluate the computational advantages of the present approach more complex geometries shall be considered in future work.

\section{Conclusions}

A so-called LFD2 method for the solution of weakly nonlinear PDEs in the frequency domain valid for arbitrary inputs has been presented. The general form has been derived and its suitability to represent nonlinear flow phenomena has been demonstrated with two application cases, one involving the Burgers' viscous equation with a nonlinear convective term and the other a quasi-one dimensional compressible unsteady flow described by the Euler equations. The method relies on the computation of the first and second order frequency-domain Volterra kernels by solving two linear algebraic systems of equations. Once the kernels have been obtained the computational effort of the nonlinear ROM is independent of the input and the problem size, as the nonlinearity dependence of the problem has been shifted outside the kernels to the functional series expansion. The method is very flexible, as apart from the general form of uni- and bidimensional convolutions for the computation of the incremental variables, a more efficient implementation to compute the incremental variables directly in the frequency domain can be used for absolutely integrable input signals. Additionally, a further simplified expression can be used if the system input consists of a multi-tone signal.

To the best of the author's knowledge this is the first time that the linear algebraic system of equations for the determination of the second order Volterra kernel has been explicitly derived for weakly nonlinear PDEs describing the compressible unsteady flow by extending the harmonic probing method previously applied to structural nonlinear systems. Even though it has been shown that a third order functional series expansion may be required for the representation of nonlinear stiffness and damping structural terms, this work shows that a second order functional series expansion may be sufficient to model weak nonlinear unsteady flow phenomena, as the nature of the nonlinearity may be regarded as caused by the convective term, which has a symmetric structure when compared with the common antisymmetric type of the structural nonlinearities.

Based on this method some further possibilities may be investigated:

- Investigation on the computational effort for the determination of the Volterra kernels of first and second order by analyzing efficient solution methods for large scale linear algebraic systems of equations. 
- Application to more complex geometries. This requires the computation of the Jacobian and Hessian matrices of the residual function, for which an automatic differentiation scheme can be used.

- Consideration of more complex flow phenomena involving for example the presence of shocks for external flow in the transonic regime.

- Extension to PDEs involving deforming grids. However, the formulation for a rigid-body motion of the grid does not require its deformation and it can be readily taken into account.

- Consideration of several inputs. In this case the Volterra cross kernels of second order have to be considered.

- Bilinear state-space formulation for the representation of the second order functional series expansion leading to a nonlinear ROM formulation in the time domain by a set of ODEs with a reduced number of degrees of freedom.

\section{References}

${ }^{1}$ C. Kaiser, D. Friedewald, D. Quero, and J. Nitzsche. Aeroelastic gust load prediction based on time-linearized RANS solutions. Deutscher Luft- und Raumfahrtkongress, Braunschweig, Germany, September 13-15 2016. https://www.dglr.de/ publikationen/2016/420161.pdf

${ }^{2} \mathrm{R}$. Thormann and M. Widhalm. Linear-frequency-domain predictions of dynamic-response data for viscous transonic flows. AIAA Journal, 51(11):2540-2557, 2013. https://doi.org/10.2514/1.J051896

${ }^{3}$ W.A Silva and R.E. Bartels. Development of reduced-order models for aeroelastic analysis and flutter prediction using the CFL3Dv6.0 code. Journal of Fluids and Structures, 19(6):729-745, 2004. https://doi.org/10.1016/j.jfluidstructs. 2004.03 .004

${ }^{4}$ D. Quero, P. Vuillemin, and C. Poussot-Vassal. A generalized state-space aeroservoelastic model based on tangential interpolation. Aerospace, 6(1):9, 2019. https://doi.org/10.3390/aerospace6010009

${ }^{5}$ A. Antoulas and D. Sorensen. Approximation of large-scale dynamical systems: an overview. International Journal of Applied Mathematics and Computer Science, 11(5):1093-1121, 2001. http://eudml.org/doc/207547

${ }^{6}$ M. Ghoreyshi, A. Jirasek, and R.M. Cummings. Reduced order unsteady aerodynamic modeling for stability and control analysis using computational fluid dynamics. Progress in Aerospace Sciences, 71:167-217, 2014. https://doi.org/10.1016/j. paerosci.2014.09.001

${ }^{7}$ K. Hall, J. Thomas, and E. Dowell. Reduced-order modelling of unsteady small-disturbance flows using a frequencydomain proper orthogonal decomposition technique. In 37th Aerospace Sciences Meeting and Exhibit, 1999. https://doi.org/ 10.2514/6.1999-655

${ }^{8} \mathrm{~T}$. Thanh and K. Willcox. Model reduction for large-scale CFD applications using the balanced proper orthogonal decomposition. 17th AIAA Computational Fluid Dynamics Conference, Toronto, Ontario, Canada, June 6-9 2005. https: //doi.org/10.2514/6.2005-4617.

${ }^{9}$ K.C. Hall, J.P. Thomas, and W.S. Clark. Computation of unsteady nonlinear flows in cascades using a Harmonic Balance technique. AIAA Journal, 40(5):879-886, 2002. https://doi.org/10.2514/2.1754

${ }^{10}$ A. Gopinath and A. Jameson. Time spectral method for periodic unsteady computations over two- and three- dimensional bodies. In 43rd AIAA Aerospace Sciences Meeting and Exhibit, 2005. https://doi.org/10.2514/6.2005-1220

${ }^{11} \mathrm{M}$. Woodgate and G. Barakos. Implicit computational fluid dynamic methods for fast analysis of rotor flows. AIAA Journal, 50(6):1217-1244, 2012. https://doi.org/10.2514/1.J051155.

${ }^{12} \mathrm{M}$. Ghoreyshi, A. Jirasek, and R. Cummings. Computational approximation of nonlinear unsteady aerodynamics using an aerodynamic model hierarchy. Aerospace Science and Technology, 28:133-144, 2013. http://dx.doi.org/10.1016/j.ast. 2012.10 .009

${ }^{13} \mathrm{M}$. Winter and C. Breitsamter. Neurofuzzy-model-based unsteady aerodynamic computations across varying freestream conditions. AIAA Journal, 54:2705-2720, 2016. https://doi.org/10.2514/1.J054892

${ }^{14} \mathrm{P}$. Bekemeyer, M. Ripepi, R. Heinrich, and S. Görtz. Nonlinear unsteady reduced-order modeling for gust-load predictions. AIAA Journal, pages 1-12, 2019. http://doi.org/10.2514/1.J057804.

${ }^{15} \mathrm{~W}$. Yao and S. Marques. Nonlinear aerodynamic and aeroelastic model reduction using a discrete empirical interpolation method. AIAA Journal, 55(2):624-637, 2017. http://doi.org/10.2514/1.J055143

${ }^{16}$ C.M. Cheng, Z.K. Peng, W.M. Zhang, and G. Meng. Volterra-series-based nonlinear system modeling and its engineering applications: A state-of-the-art review. Mechanical Systems and Signal Processing, 87(A):340-364, 2016. https://doi.org/ 10.1016/j.ymssp.2016.10.029

${ }^{17}$ W.A. Silva. Identification of nonlinear aeroelastic systems based on the Volterra theory: Progress and opportunities. Nonlinear Dynamics, 39(1):25-62, Jan 2005. https://doi.org/10.1007/s11071-005-1907-z

${ }^{18}$ W.A. Silva. Discrete-Time Linear and Nonlinear Aerodynamic Impulse Responses for Efficient CFD Analyses. PhD thesis, The College of William and Mary in Virginia, 1997.

${ }^{19} \mathrm{M}$. Balajewicz, F. Nitzche, and D. Feszty. Reduced order modeling of nonlinear transonic aerodynamics using a pruned Volterra series. 50th AIAA/ASME/ASCE/AHS/ASC Structures, Structural Dynamics, and Materials Conference, Palm Springs, CA, USA, May 4-7 2009. 
${ }^{20} \mathrm{M}$. Balajewicz and E. Dowell. Reduced-order modeling of flutter and limit-cycle oscillations using the sparse Volterra series. Journal of Aircraft, 49(6):1803-1812, 2012.

${ }^{21}$ P. Marzocca, L. Librescu, and W.A. Silva. Aeroelastic response of nonlinear wing sections using a functional series technique. AIAA Journal, 40(5):813-824, 2002. https://doi.org/10.2514/2.1735

${ }^{22}$ C.H. Lee and J.N. Juang. Nonlinear system identification - a continuous-time bilinear state space approach. The Journal of the Astronautical Sciences, 59(1-2):398-420, 2012. https://doi.org/10.1007/s40295-013-0025-2.

${ }^{23}$ K. Worden, G. Manson, and G.R. Tomlinson. A harmonic probing algorithm for the multi-input Volterra series. Journal of Sound and Vibration, 201(1):67-84, 1997. https://doi.org/10.1006/jsvi.1996.0746

${ }^{24}$ S. Liao. Homotopy Analysis Method in Nonlinear Differential Equations. 2012. http://dx.doi.org/10.1007/ 978-3-642-25132-0

${ }^{25}$ S.J. Dong, K.Z. Peng, W.M. Zhang, and G. Meng. Connection between Volterra series and perturbation method in nonlinear systems analyses. Acta Mechanica Sinica, 30(4):600-606, 2014. https://doi.org/10.1007/s10409-014-0010-4

${ }^{26}$ L.O. Chua and C.Y. Ng. Frequency domain analysis of nonlinear systems: general theory. IEE Journal on Electronic Circuits and Systems, 3(4):165-185, 1979.

${ }^{27}$ T. Hélie and M. Hasler. Volterra series for solving weakly non-linear partial differential equations: application to a dissipative Burgers' equation. International Journal of Control, 77(12):1071-1082, 2004. https://doi.org/10.1080/ 002071704200024365

${ }^{28}$ H.V. Harold and S. R. Searle. The vec-permutation matrix, the vec operator and kronecker products: a review. Linear and Multilinear Algebra, 9(4):271-288, 1981. https://doi.org/10.1080/03081088108817379

${ }^{29}$ E. Shyu and H. Caswell. Calculating second derivatives of population growth rates for ecology and evolution. Methods in Ecology and Evolution, 2014. https://doi.org/10.1111/2041-210X.12179

${ }^{30}$ T. G. Kolda. Multilinear operators for higher-order decompositions. Technical report, Sandia National Laboratories, 2006. https://doi.org/10.2172/923081

${ }^{31} \mathrm{P}$. Li and L.T. Pileggi. Compact reduced-order modeling of weakly nonlinear analog and RF circuits. IEEE Transactions on computer-aided design of integrated circuits and systems, 24(2):184-203, 2005. http://dx.doi.org/10.1109/TCAD.2004. 837722 .

${ }^{32} \mathrm{H}$. Koeppl. A local nonlinear model for the approximation and identification of a class of systems. IEEE Transactions on Circuits and Systems II: Express Briefs, 56(4):315-319, 2009. http://dx.doi.org/10.1109/TCSII.2009.2015383

${ }^{33} \mathrm{C}$. Gu. QLMOR: A new projection-based approach for nonlinear model order reduction. In 2009 IEEE/ACM International Conference on Computer-Aided Design-Digest of Technical Papers, pages 389-396. IEEE, 2009.

${ }^{34} \mathrm{H}$. Zhang and S.A. Billings. Analysing the transfer functions of nonlinear systems in the frequency domain. Research Report 445, University of Sheffield, 1992.

${ }^{35}$ R.G. Kvaternik and W.A. Silva. A computational procedure for identifying bilinear representations of nonlinear systems using Volterra kernels. Technical report, NASA Langley Research Center; Hampton, VA, United States, NASA/TM-2008215320, L-19461, 2008.

${ }^{36}$ Z.Q. Lang and S.A. Billings. Output frequency characteristics of nonlinear systems. International Journal of Control, 64(6):1049-1067, 1996. http://dx.doi.org/10.1080/00207179608921674

${ }^{37}$ L.Z. Guo, Y.Z. Guo, S.A. Billings, D. Coca, and Z.Q. Lang. A Volterra series approach to the frequency domain analysis of nonlinear viscous Burgers' equation. Nonlinear Dynamics, 70(3):1753-1765, 2012. https://doi.org/10.1007/ s11071-012-0571-3.

${ }^{38}$ J.L. Battaglia, A. Maachou, R. Malti, and P. Melchior. Nonlinear heat diffusion simulation using Volterra series expansion. International Journal of Thermal Sciences, 71:80-87, 2013. https://doi.org/10.1016/j.ijthermalsci.2013.03.016

${ }^{39}$ M.B. Giles. Nonreflecting boundary conditions for Euler equation calculations. AIAA Journal, 1989. https://doi.org/ $10.2514 / 3.10521$

${ }^{40} \mathrm{R}$. Dwight. Efficiency Improvements of RANS-Based Analysis and Optimization using Implicit and Adjoint Methods on Unstructured Grids. PhD thesis, School of Mathematics, University of Manchester, 2006.

${ }^{41}$ L.B. Rall. Automatic Differentiation: Techniques and Applications. Springer, 1981.

${ }^{42}$ U. Reif. AutoDiff toolbox. https://www.mathworks.com/matlabcentral/fileexchange/61849-autodiff_r2016b

${ }^{43}$ J. Blazek. Computational fluid dynamics: principles and applications. Elsevier, 2001.

${ }^{44}$ A. Jameson, W. Schmidt, and E. Turkel. Numerical solution of the Euler equations by finite volume methods using RungeKutta time-stepping schemes. In 14th Fluid and Plasma Dynamics Conference, 1981. https://doi.org/10.2514/6.1981-1259 